\title{
Pour une théorie économique mutualiste, profit versus surplus mutuel
}

\author{
Towards a mutualistic economic theory: Profit vs. mutual \\ surplus

\section{Por una teoría económica mutualista: lucro contra excedente} \\ mutual
}

\section{Luc Bonet}

\section{Numéro 327, janvier 2013}

URI : https://id.erudit.org/iderudit/1015148ar

DOI : https://doi.org/10.7202/1015148ar

\section{Aller au sommaire du numéro}

\section{Éditeur(s)}

Association Recma

\section{ISSN}

1626-1682 (imprimé)

2261-2599 (numérique)

Découvrir la revue

\section{Citer cet article}

Bonet, L. (2013). Pour une théorie économique mutualiste, profit versus surplus mutuel. Revue internationale de l'économie sociale, (327), 27-42. https://doi.org/10.7202/1015148ar

\begin{abstract}
Résumé de l'article
La théorie économique standard ne nous permet pas de comprendre la dynamique économique autrement que comme une recherche du profit, qui renvoie à la maximisation d'un avantage individuel. Or toute activité économique suppose un avantage mutuel. Faute d'une représentation économique correcte, celui-ci reste dans l'ombre de la théorie, alors même qu'une quantification d'un surplus mutuel est possible. Disposant d'un critère objectif, nous pouvons alors appréhender dans sa dimension critique le profit comme résultat d'une décroissance de ce surplus au long de la dynamique économique - dynamique qui dépend en dernière analyse des modes de propriété des entreprises, systèmes institutionnels fournisseurs de biens et de services. Un optimum économique peut se définir comme une situation dans laquelle l'avantage individuel des parties est maximisé pour un surplus mutuel maximum et non sous condition, totalement irréaliste, d'une concurrence " pure et parfaite ». L’analyse économique de l'avantage mutuel ouvre le champ d'une théorie économique mutualiste alternative à la théorie standard.
\end{abstract}




\section{POUR UNE THÉORIE ÉCONOMIQUE MUTUALISTE, PROFIT VERSUS SURPLUS MUTUEL}

* Responsable associatif (Centre d'information et de ressources des services à la personne [CIR-SP], Poitiers). Mél.: luc.bonet@free.fr. par Luc Bonet*

La théorie économique standard ne nous permet pas de comprendre la dynamique économique autrement que comme une recherche du profit, qui renvoie à la maximisation d'un avantage individuel. Or toute activité économique suppose un avantage mutuel. Faute d'une représentation économique correcte, celui-ci reste dans l'ombre de la théorie, alors même qu'une quantification d'un surplus mutuel est possible. Disposant d'un critère objectif, nous pouvons alors appréhender dans sa dimension critique le profit comme résultat d'une décroissance de ce surplus au long de la dynamique économique - dynamique qui dépend en dernière analyse des modes de propriété des entreprises, systèmes institutionnels fournisseurs de biens et de services. Un optimum économique peut se définir comme une situation dans laquelle l'avantage individuel des parties est maximisé pour un surplus mutuel maximum et non sous condition, totalement irréaliste, d'une concurrence "pure et parfaite ". L'analyse économique de l'avantage mutuel ouvre le champ d'une théorie économique mutualiste alternative à la théorie standard.

U

ne activité économique suppose que ses acteurs y trouvent un avantage, un bénéfice, un gain, sinon ils ne la mèneraient pas: Chacun considère que l'activité qu'il mène lui procurera une situation préférable que s'il ne la menait pas. Si cette activité (échange direct de biens et de services, coopération dans une entreprise moyennant un revenu) demande l'accord de plusieurs personnes, physiques ou morales, on doit supposer un avantage mutuel pour ces acteurs.

La théorie économique standard (TES) ne rend pas compte de celui-ci, il s'agit d'une condition nécessaire et suffisante, et cela en quelque sorte lui suffit. L'avantage mutuel est immédiatement décomposé ou analysé en avantages individuels. L'accord n'a d'autre signification économique que le signal d'un gain pour chacune des parties: une fois la transaction ou la coopération effective, chaque acteur est réputé se trouver dans une situation préférable. Pour la TES, la coopération, par rapport au système institutionnel spécifique que représente une entreprise, n'a pas de signification économique particulière, elle est assimilable à une opération d'échange: l'entreprise achète ou loue des « services producteurs » qui forment le " coût de production ». 
(1) Qui, pour l'entreprise, ne sont pas des fournisseurs de services comme les autres, la relation salariale ne s'analysant pas comme une "pure " transaction (voir la troisième partie).
Plus spécifiquement attribué à l'entreprise, le terme de profit est employé pour le revenu net positif faisant suite aux transactions avec les clients: consommateurs finaux ou entreprises qui achètent des biens intermédiaires (matières premières, machines, conseils, etc.). La différence entre le prix de vente et le coût de production détermine le profit. Cette différence est un problème critique, au sens radical du terme - une condition de possibilité qu'il faut expliciter -, son analyse se confronte à un paradoxe: si, comme le veut la théorie du marché, prix de vente et coût de production sont "donnés " à l'équilibre (tous les acteurs sont réputés "price takers", du fait de la concurrence), ce gain se fait "contre" le marché. Nous examinons ce problème théorique, et donc la notion d'équilibre, dans la première partie de cet article.

Ce faisant, nous restons dans une problématique de l'échange, mais la question de l'entreprise est elle-même critique. Il y a bien un gain spécifique à l'entreprise comme institution économique, intermédiaire entre les agents économiques classiques (clients et facteurs de production). Il constitue un problème d'un nouvel ordre par rapport à des conditions d'échange "pures »: les clients ont acheté et les fournisseurs ont été payés, les travailleurs ont reçu leur salaire, les prêteurs de capitaux ont touché leurs intérêts, il reste une espèce de gain, un "résidu ", suivant une certaine littérature économique (Perroux, 1996), qu'il faut expliquer et juger; juger suivant des critères d'efficacité distincts de ceux découlant de la théorie du marché, qui ne s'occupe que de prix.

Cela demande, d'une part, de comprendre la nature économique de la firme, pour reprendre le mot de Coase, et, d'autre part, d'analyser ses modes de propriété, qui déterminent la formation et la distribution de ce gain spécifique. Communément, le profit est jugé comme un revenu qui se fait au détriment des consommateurs ou des clients (le prix de vente pourrait être plus bas) ou au détriment des salariés ${ }^{(1)}$, soit le profit fait «au dommage d'autrui " (Montaigne).

On reconnaît là, entre autres, deux écoles critiques de l'économie dite de marché et du profit, l'une dénonçant une forme d'exploitation des consommateurs, l'autre celle des salariés; ce qui ne va pas sans contradictions dans la redistribution envisagée du profit.

Dans l'écart entre prix de vente et coût de production qui détermine le profit, mais n'en est pas nécessairement un, on peut en distinguer analytiquement deux types possibles: l'un est lié aux conditions d'échange, où la maximisation du prix de vente est visée, nous l'appellerons profit d'échange; l'autre est lié à la gestion de l'emploi salarié, où la minimisation de la masse salariale est visée, nous l'appellerons profit de gestion salariale.

Sans écarter l'intérêt et la nécessité de jugements normatifs, moraux ou politiques sur la notion de profit, on peut tenter une approche théorique basée sur la considération de l'avantage mutuel que suppose toute activité économique effective, indépendamment de ces critères normatifs, ce que demande une théorie économique à visée scientifique. 
Si l'on parle, par exemple, du profit d'une entreprise de l'industrie du luxe, il est difficile de dire que le bénéfice éventuel est un profit d'échange qui se fait au détriment des clients ou que l'entreprise en question " exploite » ses très riches clients. Parallèlement, si cette entreprise est une coopérative de production, il n'y a pas de sens à évoquer ce bénéfice comme " exploitation " des salariés, du moins à ce niveau de la production. On peut penser que la condition d'un avantage mutuel est respectée: les acteurs de l'entreprise et sa clientèle se trouvent dans une situation préférable à l'issue de leurs transactions et le bénéfice éventuel, sous forme d'une recette nette positive, participe d'un surplus mutuel global.

A celui-ci correspond une grandeur économique mesurable, une grandeur monétaire. Une analyse de ce surplus mutuel comme grandeur économique dans le cadre de l'échange est proposée dans la seconde partie de cet article. Nous analyserons ensuite cette notion dans le cadre spécifique de l'entreprise, où la relation salariale ne répond pas aux conditions de transactions "pures».

Nous disposerons alors d'un jugement sur le profit qui repose sur des critères économiques autonomes et non sur des critères normatifs, dont il faut rendre compte dans une autre sphère de valeurs (critères moraux, de justice, politiques).

\section{Notion d'équilibre économique et de profit}

(2) La notion de "recontracting" défendue par Edgeworth ouvre notamment sur la conception moderne de " marché contestable ».

(3) Pour rappel: atomicité du marché, aucun acteur ne peut imposer un prix; homogénéité des biens, même qualité des produits proposés par des entreprises concurrentes; libre entrée sur le marché, pas d'entraves pour de nouveaux acteurs; information parfaite sur la qualité et les prix; mobilité parfaite des acteurs, pas d'entraves pour aller vers les meilleurs prix.
Le monde des transactions (opérations d'échange) demande une condition de possibilité: l'autonomie des opérateurs. Ceux-ci peuvent, en tant que demandeurs ou offreurs de services, accepter ou refuser les transactions. Cette autonomie n'a de sens que si elle permet d'« aller voir ailleurs » si les prix de demande ou d'offre sont meilleurs ou encore de renégocier les prix pour qu'ils le soient ${ }^{(2)}$, ce qui suppose qu' ils peuvent l'être jusqu'à un certain point, une situation d'équilibre.

\section{Equilibre et détermination des prix}

L'équilibre économique général (EEG) a été défini comme cadre de pensée de l'économie en général par Léon Walras, à la fin du XIXe siècle. Devenu dominant comme horizon normatif pour les sciences économiques après la Seconde Guerre mondiale, il reste le cadre de pensée de toute théorie économique à nos jours.

Pour Walras, il s'agissait de fournir un modèle théorique qui rende compte de l'interdépendance des prix et en finisse avec la confusion des «classiques » sur les lois de l'offre et de la demande, comme sur le concept de "valeur ». L'état d'équilibre est la situation où le volume de biens offerts est égal au volume de biens demandés, les auteurs contemporains parlent parfois de marché "apuré ». Dans cette situation et sous condition de " concurrence pure et parfaite » (3), les prix ne peuvent être meilleurs pour les opérateurs, offreurs et demandeurs. Il y a équivalence entre l'équilibre 
(4) Vilfredo Pareto, qui a sévi de 1848 à 1923, prend la suite de Walras à l'université de Lausanne.
(5) Rien ne m'empêche de vendre mon appartement à ma nièce à un prix en dessous du marché, j'assume un coût d'opportunité dans la TES. Ce que certains analysent comme un don - nonobligation de réciprocité - n'en reste pas moins un don mesurable par rapport au marché, ce que ma nièce et moi savons. (6) Rappelons que Walras, qui a participé au mouvement coopératif, était partisan de la nationalisation des terres. offre-demande (égalité en volume) et le système de prix à l'équilibre. Cette situation d'" optimum » des prix est précisée par Pareto: on parle d'équilibre économique général quand les prix ne peuvent être meilleurs sauf à désavantager l'un au moins des opérateurs, dans le passage d'un système de prix à l'autre ${ }^{(4)}$. Dans cette situation, les prix sont des prix de marché, réputés indépendants de la volonté des opérateurs (aucun acteur économique ne peut faire jouer un "pouvoir de marché »). Les gains individuels sont maximisés sans qu'un profit puisse être dénoncé.

La différence en volume de biens entre l'offre et la demande détermine un processus d'ajustement par les prix, qui aboutit au prix de marché à l'équilibre. Il y a deux types de marché, structurellement distincts quant à la détermination des prix: un marché de biens rares (à la demande) et un marché de biens produits. Pour les biens rares, les prix augmentent jusqu'à ce que le volume de la demande s'ajuste au volume de l'offre. Pour les biens produits, l'offre s'ajuste sur le coût de production qui détermine le prix. De fait, le prix d'un bien quelconque est une combinaison de " rareté » et de « coût de production ».

\section{L'exemple de l'achat d'un bien immobilier}

Prenons un exemple: le prix du mètre carré (prix du foncier) détermine in fine le prix pour l'achat ou la location d'un bien immobilier. Concernant l'immobilier en question (l'immeuble comme bien physique), le prix d'une maison ou d'un appartement se règle sur le coût de production. Certes, des différences existent dans les coûts de construction entre la Creuse et Paris, mais elles sont bien moindres qu'entre les prix du mètre carré, prix du bien « rare » qui détermine en dernière analyse ce que l'on va appeler le marché du logement, dans la Creuse ou à Paris. Si le prix augmente dans telle ville ou tel quartier, cela signifie qu'il y a un stock de surface disponible inférieur à la demande. Le nombre de demandeurs diminue, les moins "désirants » de se loger dans telle zone et les moins fortunés des demandeurs, quelle que soit leur "désirabilité " de se loger ici préférentiellement à ailleurs, sont évincés. Le marché atteint, au fil de cette augmentation, l'équilibre de l'offre et de la demande.

Le bénéfice éventuel des propriétaires vendeurs ou loueurs d'un bien immobilier ne s'analyse pas comme un profit, mais comme un "effet d'aubaine " sur des marchés "haussiers " (effet sur lequel les spéculateurs peuvent s'appuyer). C'est cette "fatalité » du marché, en tant qu'il porte sur des biens « rares ", qui éloigne la notion de profit pour l'analyse économique standard ${ }^{(5)}$. Lorsque le prix du mètre carré baisse dans telle zone, le prix du bien immobilier s'ajuste plus précisément sur son coût de production en tant que produit et reproductible (donc comparable quant à ses qualités " physiques " intrinsèques).

En ce qui concerne les biens rares et hors intervention coopérative (publique ou privée), les prix évincent toujours les plus pauvres des demandeurs; loi du marché au sujet de laquelle Walras admet quelle puisse précisément nécessiter une intervention corrective ${ }^{(6)}$. 
Si l'on revient à la théorie « pure » et que l'on aborde le problème des prix et de l'équilibre dans le domaine de la production, c'est-à-dire quand l'offre de biens est susceptible d'augmenter avec la demande, le coût de production détermine les prix qui apurent le marché - je peux ou non acheter suivant le coût de production, et non, en théorie, en fonction de la capacité d'autrui à offrir un prix supérieur -; reste à savoir ce qu'est un " coût de production".

\section{Coût de production et extinction du profit chez Walras}

Comparé à un marché de biens rares, le marché de la production n'est confronté au problème de rationnement a priori que dans l'accès des entrepreneurs aux facteurs de production (capital et travail). Une fois les prix de ces facteurs déterminés par le marché (apuré par le volume de travail et de capital disponible confronté à la demande), le coût de production est défini, ce qui permet à Walras de déclarer: à " l'état d'équilibre de la production, les entrepreneurs ne font ni bénéfice ni perte ". La formule fera scandale chez beaucoup d'économistes, tandis que d'autres feront mine de l'ignorer, car si l'entrepreneur ne fait pas de bénéfice, pourquoi demande-t-il des services aux agents économiques propriétaires de facteurs de production? Voyons le raisonnement de Walras.

Dans la configuration "pure » d'une économie de production, nous avons pour des biens « homogènes " (de même qualité) un coût de production identique (toute entreprise étant "price taker » sur le marché des facteurs, tandis que les besoins en trésorerie doivent être pareillement estimés d'une entreprise à l'autre), d'où une concurrence qui se résume à la simple possibilité de produire les mêmes biens par des entreprises aux propriétaires distincts (notion de "marché contestable »). Pour un même volume produit vendu, cette concurrence ne peut porter que sur le bénéfice éventuel, entraînant le procès d'apurement par les prix - ici, le bénéfice comme surplus gagné sur le coût de production. Une autre entreprise propose les mêmes produits pour un moindre prix de vente et ainsi de suite jusqu'à "l'état d'équilibre de la production ", où "les entrepreneurs ne font ni bénéfice ni perte ", le prix de vente étant égal au coût de production.

Le raisonnement de Walras est en fait très élégant: l'entrepreneur ne fait pas de bénéfice ou de perte à l'équilibre de la production, mais il n'en gagne ou perd pas moins un revenu dont la maximisation est régulée par le procès d'ajustement des prix vers l'équilibre. Il ne fait aucun bénéfice comme entrepreneur, mais comme agent économique, facteur de production. Suivons Walras: à "l'état d'équilibre de la production, les entrepreneurs ne font ni bénéfice ni perte. Ils subsistent alors non comme entrepreneurs, mais comme propriétaires fonciers, travailleurs ou capitalistes dans leur propre entreprise ou dans d'autres... Et, en effet, n'est-il pas évident que s'ils retirent de leurs propres services producteurs, dans leur entreprise, un prix supérieur ou inférieur à celui qu'ils en retireraient partout ailleurs, ils sont en bénéfice ou en perte de la différence?". En bref, le bénéfice de l'entrepreneur correspond au revenu d'un facteur de production, il doit 
être comparé à ce que gagnerait l'entrepreneur comme prêteur de capital ou travailleur pour une autre entreprise. Il est maximisé quand il opère pour «son » entreprise. Walras réintègre le profit dans le coût de production et égalise par là prix de vente et coût de production: l'équilibre des marchés, avec sa condition d'une absence de profit à l'équilibre, est sauf.

\section{De la différence de rétribution des facteurs de production}

Mais le raisonnement ressemble à une habile pirouette, car il faut maintenant expliquer la différence dans le coût de production, entre cette entreprise où l'entrepreneur se fait rétribuer son "service producteur " et une autre où son service serait moins bien rétribué, plus exactement la différence de rétribution des facteurs de production et non plus des individus, suivant qui est propriétaire de l'entreprise. Cette différence n'est pas sans effet en retour sur le marché, puisque l'on peut alors imaginer que tout propriétaire d'une entreprise cherchera, comme facteur de production, à maximiser son revenu, engendrant une possible concurrence entre les agents "facteurs de production " pour la propriété des entreprises. Cela nécessite de passer de l'entrepreneur comme individu (ou groupe d'individus) qui ne dispose que d'une "idée ", comme le remarquait ironiquement Proudhon, au propriétaire d'une entreprise, système institutionnel. A l'intérieur de l'entreprise, certains, avec la capacité à vendre leur propriété ou leurs parts, sont des facteurs de production qui peuvent se payer eux-mêmes plus chers quailleurs, cette capacité étant précisément l'objet économique de la propriété; d'autres sont des facteurs de production payés au moins comme sur le marché théorique, c'est-à-dire comme dans d'autres entreprises.

Ainsi se pose une autre question d' « équilibre » entre les propriétaires, non comme individus, mais comme agents économiques distincts (demandeurs de biens produits [clients], travailleurs, prêteurs de capitaux), dont les revenus diffèrent selon leur positionnement sur le marché des transactions ou sur ce qu'il faut bien appeler le marché de la propriété d'entreprise. Or la redistribution dans le circuit économique n'est pas la même suivant les cas (revenus du capital ou revenus du travail), ce que soulignera entre autres Keynes.

\section{Evolution de la notion d'équilibre et retour du profit}

L'équilibre économique général (EEG) suppose un «état du monde » des transactions, soit les conditions d'une "concurrence pure et parfaite». Quels que soient les débats théoriques autour du modèle walrasien, le monde économique et politique réel n'est jamais dans cet état et ce n'était pas le problème de Walras dans sa recherche d'une théorie économique " pure ». L'EEG est défini comme une situation limite et théorique d'efficacité maximale de l'économie, ce qui explique à la fois la fascination qu'il exerce en tant qu'objet théorique et son importance en tant que repère rationnel quant à un optimum économique. De fait, les économistes gardent l'horizon walrasien en introduisant de l'imperfection dans 
les conditions d'échange. L'efficacité, répétons-le, est signalée par l'égalité en volume de biens offerts et demandés équivalente à un système de prix Pareto optimal. Une situation d'où le profit comme revenu de déséquilibre (inégalité entre offre et demande en volume) est exclu.

La notion va largement évoluer dans la pensée économique, à la suite de ce que l'on a appelé la "révolution keynésienne ». Pour Keynes, la crise des années 30 met en évidence un déséquilibre entre offre et demande qu'aucun ajustement par les prix ne vient résorber, le marché ne s'apure pas: déflation (les prix baissent sans que la consommation reprenne) et chômage "involontaire " (des salariés ne trouvent pas de travail quand bien même ils acceptent les salaires offerts) signent cette crise. Le processus d'ajustement de l'offre et de la demande par les prix ne fonctionne tout bonnement pas et, pour Keynes, la baisse des salaires, solution théorique classique (l'offre est trop importante, son prix doit baisser pour trouver une demande), ne ferait qu'empirer les choses, en déprimant encore plus la demande.

Avec les années 70, l'éclat de la révolution keynésienne commence à pâlir quand inflation et chômage vont de pair. Outre une contestation toujours plus formelle des thèses keynésiennes et un progrès technique dans la théorie des modèles, on assiste à une contre-offensive méthodologique (la manière d'appréhender une situation), qui remet en avant l'école ultra-libérale du laisser-faire.

\section{"Discipline d'équilibre " et raisonnement circulaire}

L'exigence d'une "discipline d'équilibre " pour aborder tout problème économique est notamment mise en avant par Robert Lucas, étoile montante de cette école ultralibérale, discipline à laquelle aurait dérogé Keynes, notamment dans son approche du chômage, que Lucas se refuse à définir comme "involontaire " et qui correspond à "la contrainte de construire la théorie économique sur la base des postulats de comportement optimisateur et d'apurement des marchés " (De Vroey, 2009).

Il s'agit, pour Lucas, de retourner à la réalité via un recours au raisonnement walrasien d'équilibre de l'offre et de la demande par l'ajustement des prix, un recours paradoxal. Lucas intègre les progrès de la théorie des modèles en général, en dynamisant la notion d'équilibre (dimension stochastique de tout système), mais son originalité comme théoricien tient à ce qu'il allège de toute visée d'efficacité maximale le jugement économique: dès lors qu'une économie est décentralisée et qu'il existe plusieurs centres de décision relativement à la production et à l'acquisition de biens, on doit postuler à tout moment un apurement des marchés; le monde réel est celui des transactions effectives (des échanges ont effectivement lieu) bousculé par des "chocs" (dynamique stochastique, soit aléatoire, du système) auxquels les agents économiques répondent par des stratégies (dimension intertemporelle de l'anticipation rationnelle des acteurs).

Evaluer l'efficacité de ces transactions effectives selon une situation d'équilibre économique général n'a pas de sens pour Lucas. "Vous pouvez 
(7) C'est ce qui a entraîné les critiques des ultralibéraux sur le système de protection sociale trop généreux. regarder par la fenêtre et vous demander si la Nouvelle-Orléans est à l'équilibre. Qu'est-ce que cela pourrait bien signifier? L'équilibre est une caractéristique de la manière dont nous regardons les choses et non de la réalité " (De Vroey, 2009).

Derrière cette attitude (ce "regard »), il y a une utilisation de la notion d'équilibre très différente de celle qui animait Walras. Ici, par « discipline d'équilibre ", on doit postuler que chaque partie est gagnante dans la transaction (comportement optimisateur), sinon elle ne la conclurait pas (autonomie), et si elle ne la conclut pas, elle ne peut être dite perdante, sinon elle accepterait la transaction (comportement optimisateur). C'est un raisonnement circulaire d'où toute notion de perte ou de moindre efficacité est exclue, mais aussi un renversement total quant à la perspective sur le profit. Dans la mesure où Lucas considère que le volume de biens, à l'offre comme à la demande, est exactement celui qui est échangé à tout moment sur le marché des transactions, le système économique est toujours à l'équilibre, en un sens walrasien. La notion de perte économique, qui suppose une inégalité en volume de biens à l'offre et à la demande avant que les prix d'équilibre ne soient trouvés, n'a pas de pertinence et le profit est un revenu qui signale non seulement le succès de telle ou telle transaction, mais aussi la bonne santé du système économique en général: le profit ne se fait jamais « contre » le marché. Il n’y a, par exemple, pas de chômage que l'on puisse définir comme involontaire, il y a seulement "l'effet de choix individuels " où des individus font le choix du « loisir » faute de réaliser un profit en étant salariés ${ }^{(7)}$.

C'est la raison pour laquelle il n'est pas inutile de garder sous la main une perspective d'équilibre avec son souci d'une efficacité maximale et son intuition d'une perte économique possible, quitte à s'éloigner de Walras, mais dans un autre sens que nos modernes théoriciens du laisser-faire.

\section{Echanges et surplus mutuel}

Imaginons que Julie a en sa possession un objet B dont elle est disposée à se séparer pour la somme de 30 euros, pas moins. Julie compte vendre $\mathrm{B}$ à l'occasion d'un vide-grenier. Idriss, de son côté, envisage de faire un cadeau pour lequel il ne veut pas dépenser plus de 50 euros. Il se rend au vide-grenier dans cette intention.

\section{Une grandeur économique pour l'avantage mutuel lié à l'échange}

Idriss est intéressé par B et Julie et lui s'accordent sur un prix de 35 euros (prix affiché arbitrairement par Julie, révisable par elle dans le déroulement de la journée de vente ou prix résultant d'un marchandage). Relativement à son prix minimum de cession (30 euros), on peut dire que Julie réalise un bénéfice de 5 euros. Si l'on s’appuie sur le prix maximum qu'Idriss était prêt à payer (50 euros), on peut dire qu'achetant à 35 euros il réalise un gain de 15 euros. On parle ainsi de "surplus 
(8) Le terme ayant été introduit dans la théorie économique par Marshall.

(9) De façon rigoureuse, une infinité, la monnaie étant une grandeur continue. De la même manière, on peut dire qu'ily a quantité de situations Pareto optimales, le critère de Pareto n'a d'intérêt que dans la situation purement abstraite d'équilibre économique général, ce qui ne l'empêche pas d'être utilisé à tort et à travers dans la littérature économique.

(10) Mais qui est contestable sous un aspect objectif: les consommateurs, même les plus aisés, ne cherchent pas nécessairement les prix les plus bas, par exemple. (11)L'intérêt de la notion de "surplus " a été rigoureusement examiné par Maurice Allais, sans que son ambition théorique soit reprise paruneécole (Allais, 1981). du consommateur" (Dupuit, 2009 $\left.{ }^{(8)}\right)$. Le bénéfice de Julie que l'on peut appeler «surplus du vendeur » et le «surplus du consommateur » d'Idriss augmentent individuellement le pouvoir d'achat de chacun.

La transaction libère un surplus monétaire de 20 euros (le gain de Julie de 5 euros auquel s'ajoute le gain d'Idriss de 15 euros). Celui-ci, comme résultat économique de l'échange effectif, est indépendant de la répartition des gains et donc de leur maximisation individuelle: Idriss était prêt à acheter à 50 euros, Julie aurait alors gagné 20 euros par rapport à son prix minimum de cession (ou offre); Julie était prête à vendre à 30 euros, Idriss aurait gagné 20 euros relativement à son prix maximum d'achat (ou demande). Le surplus monétaire de cet échange spécifique peut être qualifié de "surplus mutuel ", une grandeur économique qui correspond à l'avantage mutuel de la transaction. Cet avantage mutuel explique que cette dernière puisse être effectivement tenue. Lorsque nous achetons ou vendons un bien ou un service 30 euros, nous n'échangeons pas 30 euros contre 30 euros.

Il y a, dans ce cas de transaction spécifique, un ensemble de prix possibles ${ }^{(9)}$ pour les acteurs, mais un même "surplus mutuel ». Nous avons une grandeur économique en cohérence avec la notion d'avantage mutuel comme condition et résultat d'un échange, susceptible de varier positivement ou négativement et donc de mesurer une efficacité économique plus ou moins grande - évaluation indépendante du problème de la maximisation du gain individuel des opérateurs.

Lorsque l'on s'en tient au principe d'une maximisation du gain individuel dans l'échange, le prix "efficace " pour l'échange effectif entre Julie et Idriss est indécidable, chaque prix correspondant à une situation Pareto optimale: si l'on passe d'un prix de 35 euros à 40, Julie est avantagée, mais Idriss perd 5 euros. C'est cette indétermination que le marché est censé lever, d'un point de vue théorique, en permettant de confronter l'ensemble des offreurs et des demandeurs pour un résultat final où le prix est strictement déterminé: c'est la situation d'équilibre, où les acteurs sont réputés "preneurs de prix ", dite d'efficacité économique maximale. Mais cette indétermination n'en est une seulement si l'on pose que, la maximisation du gain individuel étant l'objectif de chaque opérateur économique (ce que nous ne contestons pas sous un aspect " subjectif » ${ }^{(10)}$ ), il s'en suit une situation d'efficacité économique maximale équivalente à un maximum de gain individuel. Pour y voir plus clair, revenons à une économie de production (entreprises-clients), pour suivre l'évolution possible $\mathrm{du} \ll$ surplus mutuel $»^{(11)}$.

\section{Le profit comme décroissance du surplus mutuel}

Dans une économie de production où une entreprise produit un même bien à un prix unique pour une pluralité de clients, chaque client est doté d'un pouvoir d'achat et d'une "désirabilité » de ce bien qui déterminent un "surplus du consommateur" (supérieur ou égal à zéro) propre à chacun: le coût n'est pas le même pour deux acheteurs 
(12) Cette perspective sur les transactions économiques est due à un ingénieur français du $\mathrm{XIX}$ siècle, Jules Dupuit, dont deux mémoires sont restés marquants dans l'histoire de la pensée économique, sans être pour autant utilisés au mieux (De la mesure de l'utilité des travaux publics [1844] et De l'influence des péages sur l'utilité des voies de communications [1849]). d'un même bien au même prix suivant leur pouvoir d'achat (paramètre qui influe la "désirabilité " elle-même). Une dépense de 100 euros me coûte plus si je gagne 1000 euros par mois que si j'en gagne 5000 . Ce coût, qui apparaît comme subjectif, est précisément mesuré par le prix qui me pousse à renoncer à l'achat ou à le rationner dans le cas d'une grandeur continue, soit lorsque mon "surplus du consommateur " s'annule quand le prix de vente augmente. Le renoncement à l'acquisition détermine un coût objectif ${ }^{(12)}$.

\section{L'exemple de l'entreprise $J$}

Supposons que l'entreprise Julie $(\mathrm{J})$ produise cent unités d'un bien B (ou mille ou un million), pour lesquels elle demande 500 euros par unité. Admettons qu'avec la vente de ce volume J couvre exactement son coût de production, qui comprend le revenu du propriétaire, jugé suffisant pour l'inciter à produire: J se trouve alors en situation d'équilibre.

Pour simplifier, imaginons qu'il y ait cent acquéreurs (l'achat est d'une unité de $\mathrm{B}$ ou de zéro). Comme nous ne connaissons pas le prix maximum qu'est prêt à payer chacun des acquéreurs, soit le «surplus du consommateur" pour chaque client qui acquiert B au prix demandé, le «surplus mutuel » pour l'ensemble des acteurs (J et ses clients) n'est pas calculable précisément, mais nous savons qu'il est maximum, le volume produit étant intégralement vendu au prix unitaire minimum déterminé par le coût de production de l'entreprise J. L'ensemble des acteurs se situe dans une situation préférable du fait de cette transaction que sans elle.

Imaginons qu'au prix de 600 euros, avec les mêmes clients (il n'y a pas de raison économique que de nouveaux clients apparaissent, ils n'achetaient pas à 500 euros) et pour un coût de production identique, J vende aussi bien ses cent unités : l'entreprise fait un bénéfice relatif, « surplus du vendeur ». En se rapportant à la situation antérieure, on peut considérer que du côté des clients il y a une perte globale du même montant, mais le «surplus mutuel » pour cette transaction demeure identique, le bénéfice de J compense le moindre gain des clients et nous avons le même "surplus mutuel ", différemment distribué, mais toujours maximum.

Supposons que pour une augmentation du prix au-delà de 600 euros un certain nombre de consommateurs ou clients renoncent à l'achat (les moins "désirants " du bien et toujours les moins aisés de la population en cause): le volume de vente baisse, tandis que le bénéfice du vendeur augmente, la perte de recette du fait des clients "évincés " étant plus que compensée par le nouveau prix que les clients restants sont encore disposés à payer. Supposons que le vendeur augmente encore son prix, le nombre de clients disposés à acheter plus cher diminue. Le bénéfice du vendeur croît jusqu'à un point d'inflexion maximum à partir duquel ce bénéfice s'atténue (trop peu d'acheteurs désormais au nouveau prix), puis s'annule.

Relativement aux conditions de la transaction à 600 euros, des clients ont été évincés par le nouveau prix, le gain pour les clients restants est moindre, 
(13) Suivant les ruses du marketing qui visent à déshomogénéiser les biens offerts, "[...] car on n'est jamais dupe que par rapport aux frais de production. L'acheteur ne paye jamais un produit au-delà de la valeur d'utilité qu'il y attache " (Dupuit, 1844). mais on peut toujours le considérer comme compensé par le bénéfice en augmentation de J. Le « surplus mutuel » de ce côté-là (partiel) reste d'un même montant, il manque par contre au " surplus mutuel » total le montant des achats des clients évincés. Dans cette nouvelle transaction, le "surplus mutuel » a baissé, on peut objectivement juger d'une moindre efficacité économique pour cette opération spécifique: une perte économique d'une situation à l'autre.

Cette démonstration est en phase avec le raisonnement walrasien d'équilibre comme égalité de volume produit et vendu: quand des clients renoncent à l'achat, il reste pour cet échange un stock de biens produits qui constitue une perte "sèche " pour l'ensemble des opérateurs (situation de déséquilibre économique), mais, dans la vie économique réelle, l'existence provisoire de ce stock n'affecte pas l'entreprise, qui ajuste sa production sur la maximisation de son bénéfice et non sur le coût de production.

Reste la possibilité de biens de substitution, disons $\mathrm{C}$, pour les clients évincés. Ce bien $\mathrm{C}$ doit être supposé de moins bonne qualité( ${ }^{(13)}$ pour un prix inférieur à 600 euros si tel n'était pas le cas, J serait obligée de revenir à son prix de vente de 500 euros, puisque ses autres clients achèteraient eux aussi $\mathrm{C}$ (d'où un calcul possible de la perte pour un prix de $\mathrm{C}$ compris entre 500 et 600 euros, soit la différence que les clients sont obligés de payer pour un bien de substitution, du fait de l'augmentation décidée par l'entreprise J).

\section{Dépasser une critique morale de l'économie}

La théorie économique standard considère que l'entreprise, dont la fonction objectif serait par définition la maximisation du bénéfice, est naturellement amenée à augmenter son prix jusqu'au point d'inflexion qui détermine le maximum de bénéfice et que ce dernier est le signe d'une efficacité économique maximale. Une analyse économique que l'on peut qualifier de mutualiste - basée sur l'analyse des transactions comme devant dégager un «surplus mutuel » maximum pour une efficacité maximale considérera que l'augmentation du prix entraînant, à un certain point, le renoncement à l'achat de clients éloigne la transaction d'une efficacité économique maximale: à partir d'un certain prix, une fraction du bénéfice devient profit et signifie une perte économique, un moindre avantage mutuel objectivement évalué par un "surplus mutuel » en baisse.

Nous avons donc un critère économique " pur » à disposition pour juger de la différence entre bénéfice et profit. Cette grandeur, le «surplus mutuel ", nous permet d'avoir un jugement sur l'efficacité économique indépendant de critères normatifs extérieurs à la sphère économique. Cela ne veut pas dire que ces critères normatifs, quels qu'ils soient, ne soient pas nécessaires et toujours présents dans la vie d'une société, mais qu'ils ne peuvent à eux seuls nous permettre de nous orienter dans la pensée économique, ce que demande une critique de l'économie qui ne se contente pas d'être une critique de "belles âmes". 


\section{Surplus mutuel et relation salariale}

La relation salariale au sein d'une entreprise ne peut s'analyser comme une opération d'échange, une transaction. Les salariés ne sont pas des fournisseurs dont l'entreprise acquiert les "services producteurs " dans les mêmes conditions que pour les autres services. Le travail coopératif qu'implique le procès de production demande une coordination de l'activité qui ne peut s'expliquer par le mécanisme des transactions. C'est ce que remarque et développe Ronald Coase dans un article de 1937, "La nature de la firme ": "En dehors de l'entreprise, les mouvements des prix dirigent la production, laquelle se voit coordonnée à travers une série de transactions intervenant sur le marché. Au sein de l'entreprise, [celles-ci] sont éliminées et l'on substitue à la structure compliquée du marché et de ses transactions d'échange l'entrepreneur coordinateur qui dirige la production. " La relation salariale, de subordination entre employeur et employé, ne peut s'expliquer par l'échange marchand qui demanderait que l'employé soit "indépendant", "une personne engagée uniquement pour remettre à l'employeur les fruits de son travail ».

\section{Une subordination hiérarchique indépendante du mode de propriété} Pour notre auteur, la logique de marché - qui implique "que les hommes, agissant comme des individus totalement libres, mais sans entente aucune, ont organisé une vie économique avec une division du travail primaire et secondaire, utilisation du capital, etc. " (Knight, cité par Coase) - ne peut s'appliquer à la firme, elle ne l'explique pas. Si la théorie économique substitue aux « individus totalement libres » des termes plus neutres comme "unités économiques ", la perspective reste la même: le marché est bien une activité des " unités économiques " coordonnée par les prix et "sans entente aucune", ce qui est précisément impensable pour l'activité au sein de l'entreprise.

Dès lors, nous dit Coase, on doit définir la firme comme "un système de relations qui apparaît quand la répartition des ressources dépend d'un entrepreneur ". Cette solution présente un avantage mutuel pour les travailleurs et l'entrepreneur, seuls acteurs économiques concernés par cet accord, qui fonde en retour le système institutionnel particulier qu'est l'entreprise, les autres facteurs (biens intermédiaires, y compris le capital) étant fournis par le marché. Ce système institutionnel est bien une activité économique particulière, distincte d'une opération d'échange marchand, la différence portant sur la coopération qui dégage un surplus mutuel.

L'avantage mutuel porte sur la gestion de l'emploi: la coordination hiérarchique diminue pour chaque acteur le coût de cette gestion, soit le coût qu'il devrait supporter pour la série de contrats à conclure et juxtaposer dans une activité collective qui ne serait pas coopérative (" sans entente aucune »). Nous n'avons pas un marché du travail « libre », mais 
(14) Pour une discussion sur ce "préjugé " chez Coase, voir Bonet, 2010. un marché de l'emploi, la pluralité des firmes induisant la formation des salaires pour lesquels les entreprises sont "price takers".

Il y a donc pour et par la firme un coût d'organisation de la production inférieur à celui des transactions pour une même production. En tant qu'activité, cette organisation s'analyse comme un travail, et il lui correspond, en termes de revenu, un salaire, celui d'organisateur de l'emploi dans la firme.

La théorie économique n'induit rien de plus et notamment pas que le gérant, ou le manager, doit être propriétaire de la firme, la personne physique ou morale qui décide en dernier ressort de la répartition des ressources: la subordination hiérarchique ne préjuge pas du mode de propriété $^{(14)}$. Cette distinction est suffisamment compréhensible quand on examine le cas des coopératives de production aux mains des travailleurs: la répartition des ressources est en théorie décidée par le collectif de travail. Cette distinction entre propriétaire et gestionnaire de l'emploi trouve sa place dans la pensée économique suite à l'analyse des grands groupes capitalistes où l'entrepreneur dit traditionnel (travaillant et contrôlant la production) s'efface devant un actionnariat extérieur au travail et au contrôle de la production: dans la relation d'agence, qui distingue un "principal " et un "agent ", l'agent optimise la production pour le compte du principal. La question sur le statut économique de ce dernier - statut que ne détermine en rien la forme vide de la propriété, celle-ci demandant une mutation entre une fonction d'agent économique du marché et la position de propriétaire - demeure: dans l'entreprise capitaliste, les prêteurs de capitaux, offreurs sur le marché des services, "deviennent " propriétaires; dans l'entreprise coopérative, les travailleurs, offreurs sur le marché de l'emploi, "deviennent " propriétaires; dans l'entreprise publique ou mutualiste, les clients (usagers, consommateurs, citoyens, etc.), demandeurs sur le marché des biens et des services, "deviennent " propriétaires. Ce « devenir " propriétaire trouve davantage son explication dans l'histoire politique et sociale que dans la pensée économique standard. Il n'en reste pas moins que la gestion du salariat sera a priori différente suivant l'agent économique propriétaire de la firme.

\section{Profit de gestion salariale}

Le surplus mutuel relatif à la coopération entrepreneuriale s'analyse monétairement comme la masse salariale répartie suivant la gestion de l'emploi (rappelons que le salaire est le taux temps de travail par salaire horaire).

Relativement à une entreprise, pour une recette donnée (supérieure ou égale à zéro) et un même système de prix comme une même solution technique - soit une situation d'équilibre pour l'entreprise -, le revenu du propriétaire est maximisé en minimisant la masse salariale.

Cette baisse de la masse salariale est compatible avec l'augmentation de salaire individuel pour certains secteurs au sein de l'entreprise, 
notamment le management. Elle entraîne un processus d'ajustement de l'emploi, s'analysant comme un profit, puisqu'il dégrade la situation de certains acteurs de la relation salariale rapportée à la situation antérieure d'équilibre. Nous avons ici un problème de redistribution à la fois plus classique et plus complexe que dans la relation d'échange: d'une part, au sein de la population des travailleurs liés à l'entreprise, certains peuvent voir ou non leur salaire augmenter, tandis que pour d'autres il diminue nécessairement (paramètre temps de travail); d'autre part, le profit (revenu issu de la baisse globale de la masse salariale) est redistribué vers le propriétaire de l'entreprise, que l'on doit comprendre comme agent économique, c'est-à-dire les utilisateurs du bien produit, les prêteurs de capitaux ou les travailleurs comme propriétaires. Dans ce dernier cas (coopérative de production), le profit de gestion qui dégrade la situation de certains travailleurs n'est pas envisageable, au moins en théorie, dans le cadre démocratique d'un groupe de pairs.

\section{Conclusion}

Sans autre considération pour l'avantage mutuel comme condition à toute activité économique demandant l'accord des parties, la théorie économique standard s'appuie sur une représentation particulière de l'échange qu'elle généralise à l'ensemble des activités économiques: dynamique de marchandage entre deux acteurs, situation indécidable sur la fixation d'un prix qui maximiserait l'avantage individuel. La solution se trouve dans l'exploration de l'ensemble de ces transactions possibles (le marché) donnant une solution finale qui fixe les prix pour tous et par conséquent les limites de cette maximisation individuelle.

Dans le secteur économique de la production, les fournisseurs de services sont les entreprises. Chacune d'entre elles s'adresse à un nombre indéfini de demandeurs, aux "désirabilités " et aux revenus différents, d'où une pluralité de parties qui ne se laisse pas analyser comme une juxtaposition d'échanges "deux à deux ", mais bien plutôt comme un offreur qui s'adresse à des groupes sociaux dont le paramètre décisif et distinctif est en dernier lieu leur pouvoir d'achat. Cet " état du monde " réel détermine, pour une transaction donnée entre la firme et ses clients, un surplus mutuel, grandeur mesurable qui varie suivant les choix de prix de vente effectués par la firme - choix de prix que permet la simple différenciation d'avec un produit concurrent, et qui du coup ne l'est plus rigoureusement. On peut identifier un profit d'échange quand, d'une part, ce prix augmente sans que le coût de production progresse dans les mêmes proportions et quand, d'autre part, des clients sont alors évincés ou rationnés: le surplus mutuel diminue.

La relation salariale, que la TES présente elle aussi comme un échange, demande une relation de coordination hiérarchique au sein de l'entreprise, relation qui présente un avantage mutuel pour les parties. Relativement 
au procès de production, cette coordination est technique (organisation) et politique (commandement-obéissance), ce que la TES ne peut analyser comme phénomène économique en se bornant au "mouvement des prix » qui expliquerait la coordination de l'activité " sans entente aucune » entre " unités économiques ». Comme phénomène économique, la firme se distingue de la dynamique des transactions par le pouvoir du propriétaire sur « la répartition des ressources » de l'entreprise et un pouvoir sur la gestion de l'emploi déterminé donc par l'intérêt économique des propriétaires des firmes. On peut identifier un profit de gestion quand, pour une recette supérieure ou égale à zéro, le gestionnaire des ressources - propriétaire de la firme comme décideur en dernier ressort - obtient une baisse de la masse salariale, variable du surplus mutuel quantifiable dans la relation salariale.

Pour la TES, la fonction objectif de l'entreprise est le profit, alors qu'une représentation réaliste de l'entreprise qui prend en compte l'ensemble des modes de propriété de la firme existant ou possible (entreprises publiques, mutualistes, capitalistes, coopératives, etc.) n'indique qu'une fonction objectif commune à toute firme: l'absence de pertes, conditions de la continuité de son activité économique. On remarquera qu'en privilégiant en tant que référence théorique la firme capitaliste, la TES désigne comme modèle un type particulier d'entreprise qui, dans la dynamique économique, cumule les deux possibilités de profit et donc de perte pour une population donnée: profit d'échange (" contre» les clients-usagers) et profit de gestion (" contre » les salariés). On peut y voir une explication assez robuste du "paradoxe de la pauvreté dans l'abondance " que soulignait Keynes dans les années 30 et dont l'actualité ne se dément pas.

Ce faisant, la TES ne développe pas une théorie économique réaliste fondée sur le réquisit à toute activité économique, l'avantage mutuel, cette mutualité de l'avantage devant être analysée dans les relations d'échange et de coopération, distinguées et situées dans leurs domaines respectifs, soit une théorie économique mutualiste restant à fonder hors des champs normatifs qui lui tiennent habituellement lieu de référence. 


\section{Bibliographie}

Allais M., 1981, La théorie générale des surplus, Presses universitaires de Grenoble.

Dupuit J., 2009, Euvres complètes, vol. I, Economica.

Bonet L., 2010, "Proudhon-Coase : la pro- Francois P., 2008, Sociologie des marchés, priété de la firme », Recma, n 317. Armand Colin.

Coase R., 2005, L'entreprise, le marché et le droit, recueil d'articles, traduit et présenté par Boualem Aliouat, Editions d'organisation. De Vroey M., 2009, Keynes, Lucas, d'une macroéconomie à l'autre, Dalloz.

Perroux F., 1996, Le problème du profit, Presses universitaires de Grenoble.

Walras L., 1988, Eléments d'économie politique pure, vol. VIII des œuvreséconomiques complètes d'Auguste et Léon Walras, Economica. 